Bull. Korean Math. Soc. 51 (2014), No. 6, pp. 1579-1589

http://dx.doi.org/10.4134/BKMS.2014.51.6.1579

\title{
A NOTE ON GENERALIZED DIRAC EIGENVALUES FOR SPLIT HOLONOMY AND TORSION
}

\author{
Ilka Agricola And Hwajeong Kim
}

\begin{abstract}
We study the Dirac spectrum on compact Riemannian spin manifolds $M$ equipped with a metric connection $\nabla$ with skew torsion $T \in \Lambda^{3} M$ in the situation where the tangent bundle splits under the holonomy of $\nabla$ and the torsion of $\nabla$ is of 'split' type. We prove an optimal lower bound for the first eigenvalue of the Dirac operator with torsion that generalizes Friedrich's classical Riemannian estimate.
\end{abstract}

\section{Introduction}

It is well-known that for a Riemannian manifold $(M, g)$, the fact that the holonomy representation of the Levi-Civita connection decomposes in several irreducible modules has strong consequences for the geometry of the manifold - by de Rham's theorem, the manifold is locally a product, and the spectrum of the Riemannian Dirac operator $D^{g}$ can be controlled ([7], [21]).

If the Levi-Civita connection is replaced by a metric connection with torsion, not much is known, neither about the holonomy nor about the implications for the spectrum. This note is a contribution to the much larger task to improve our understanding of the holonomy of metric connections with skew-symmetric torsion. The foundations of the topic were laid in [4] (see also the review [2]), substantial progress on the holonomy in the irreducible case was achieved in [26] and [25]. If the connection $\nabla$ is geometrically defined, that is, it is the characteristic connection of some $G$-structure on $(M, g)$, one is interested in the spectrum of the associated characteristic Dirac operator $\not D$, a direct generalization of the Dolbeault operator for a hermitian manifold and Kostant's cubic Dirac operator for a naturally reductive homogeneous space. In [6], it was outlined that the first eigenvalue of $D D$ may be estimated from below if the

Received May 22, 2013.

2010 Mathematics Subject Classification. 53C25, 53C26, 53C27, 53C28, 53C29, 58J50, $58 \mathrm{~J} 60$.

Key words and phrases. Dirac operator, eigenvalue estimate, metric connection with torsion.

This work was supported by NRF(National Research Foundation of Korea) Grant funded by the Korean Government(NRF-2010-0002925) and Hannam University Research Fund in 2014 . 
torsion is parallel; however, the paper could only deal with $G$-structures on a case by case basis. A first general eigenvalue estimate depending only on the connection $\nabla$ was given by means of twistor theory in the authors' joint paper [3] with J. Becker-Bender. In this paper, we also examined the case that the manifold was reducible, that the holonomy of the connection $\nabla$ decomposed accordingly, and that the torsion 3 -form had no mixed parts.

This note is the first article devoted to the situation that the holonomy representation of $\nabla$ on $T M$ splits into several irreducible submodules, but the underlying manifold can yet be irreducible. We can then prove an optimal eigenvalue estimate (Corollary 2.1) for the Dirac operator $\not D$ under the assumption that the torsion has no non-trivial contribution on any of the $\nabla$-parallel distributions of $T M$ - we call $(M, g, \nabla)$ then a manifold with split holonomy (see Definition 2.1). Examples show that such geometries arise quite naturally in the investigation of $G$-structures, a fact that had not been observed before.

\section{The estimate}

\subsection{Geometric set-up}

We assume that $\left(M^{n}, g\right)$ is an oriented Riemannian manifold endowed with a metric connection $\nabla$ with skew-symmetric torsion $T \in \Lambda^{3}\left(M^{n}\right)$,

$$
\nabla_{X} Y:=\nabla_{X}^{g} Y+\frac{1}{2} \cdot T(X, Y,-) .
$$

The holonomy group $\operatorname{Hol}\left(M^{n} ; \nabla\right)$ is then a subgroup of $\operatorname{SO}(n)$, and we shall assume that it is a closed subgroup to avoid pathological cases. In order to distinguish it from the torsion, the tangent bundle and its subbundles will be denoted by $\mathcal{T} M^{n}, \mathcal{T}_{1}, \mathcal{T}_{2} \ldots$. Recall that for a $\nabla$-parallel distribution, the standard proof of the following basic lemma carries over from Riemannian geometry without modifications (see for example [23, Prop. 5.1]).

Lemma 2.1. Let $\mathcal{T} \subset \mathcal{T} M^{n}$ be a parallel distribution and $Y \in \mathcal{T}$. For any $X \in \mathcal{T} M^{n}, \nabla_{X} Y$ is again in $\mathcal{T}$; in particular, $R\left(X_{1}, X_{2}\right) Y \in \mathcal{T}$ for any $X_{1}, X_{2}$.

Let $\mathcal{T}$ be a parallel distribution, $\mathcal{N}$ its orthogonal distribution defined by $\mathcal{N}_{x}:=\mathcal{T}_{x}^{\perp}$ in every point $x \in M^{n}$. The fact that all elements of $\operatorname{Hol}\left(M^{n} ; \nabla\right)$ are orthogonal transformations implies that $\mathcal{N}$ is again a parallel distribution. Thus, the tangent bundle splits into an orthogonal sum of parallel distributions $\left(n_{i}:=\operatorname{dim} \mathcal{T}_{i}\right)$

$$
\mathcal{T} M^{n}=\mathcal{T}_{1} \oplus \cdots \oplus \mathcal{T}_{k} \text {, and } \operatorname{Hol}\left(M^{n} ; \nabla\right) \subset \mathrm{O}\left(n_{1}\right) \times \cdots \times \mathrm{O}\left(n_{k}\right) \subset \mathrm{SO}(n) .
$$

We assume that every distribution $\mathcal{T}_{i}$ is again orientable and that the holonomy preserves the orientation, i.e., we assume

$$
\operatorname{Hol}\left(M^{n} ; \nabla\right) \subset \mathrm{SO}\left(n_{1}\right) \times \cdots \times \mathrm{SO}\left(n_{k}\right) .
$$

We denote an orthonormal frame of $\mathcal{T}_{i}$ by $e_{1}^{i}, \ldots, e_{n_{i}}^{i}, i=1, \ldots, k$. For convenience, we assume that the spaces $\mathcal{T}_{i}$ are numbered by ascending order, 
$n_{1} \leq n_{2} \leq \cdots \leq n_{k}$. We recall the following properties of the curvature of the connection $\nabla$ from our previous article [3]:

(1) Since the distributions $\mathcal{T}_{i}, \mathcal{T}_{j}$ are orthogonal, Lemma 2.1 implies for any vector fields $X, Y$ that $g\left(\mathcal{R}(X, Y) \mathcal{T}_{i}, \mathcal{T}_{j}\right)=0$ if $i \neq j$.

(2) The Ambrose-Singer theorem implies that the curvature operator $R(X, Y)$ vanishes if $X \in \mathcal{T}_{i}, Y \in \mathcal{T}_{j}, i \neq j$.

(3) The Ricci tensor has block structure,

$$
\text { Ric }=\left[\begin{array}{c|c|c}
\operatorname{Ric}_{1} & 0 & \\
\hline 0 & \ddots & 0 \\
\hline & 0 & \operatorname{Ric}_{k}
\end{array}\right],
$$

i.e., $\operatorname{Ric}(X, Y) \neq 0$ can only happen if $X, Y \in \mathcal{T}_{i}$ for some $i$.

(4) The scalar curvature splits into 'partial scalar curvatures' $\mathrm{Scal}_{i}:=$ $\operatorname{tr} \mathrm{Ric}_{i}$, and Scal $=\sum_{i=1}^{k} \mathrm{Scal}_{i}$.

Be cautious that despite of the block structure of the Ricci curvature, one has in general that $R(X, Y, U, V) \neq 0$ if $X, Y \in \mathcal{T}_{i}, U, V \in \mathcal{T}_{j}$ for $i \neq j$. The space of 3 -forms splits under the holonomy representation into

$$
\Lambda^{3}(\mathcal{T})=\bigoplus_{i=1}^{k} \Lambda^{3}\left(\mathcal{T}_{i}\right) \oplus \bigoplus_{i \neq j} \Lambda^{2}\left(\mathcal{T}_{i}\right) \wedge \mathcal{T}_{j} \oplus \bigoplus_{i<j<k} \mathcal{T}_{i} \wedge \mathcal{T}_{j} \wedge \mathcal{T}_{k}
$$

In our first paper [3], we treated in detail the situation that the torsion $T$ of the connection $\nabla$ is entirely contained in the first summand, i.e., may be written as a sum $T=\sum_{i} T_{i}$ with $T_{i} \in \Lambda^{3}\left(\mathcal{T}_{i}\right)$. This is basically the case when $M$ is locally a product.

The main point of this note is the observation that the other extreme case, i.e., that $T$ consists only of terms of the third type, can also be controlled and is in fact not so exotic as it may appear. Examples will be given in the last section. Thus, we define:

Definition 2.1. If the torsion $T$ satisfies $T(X, Y)=0$ whenever $X, Y \in \mathcal{T}_{i}$ and $\nabla T=0$, we shall call $(M, g, \nabla)$ a manifold with split holonomy.

Although the definition would make sense without the additional assumption $\nabla T=0$, we shall see in the sequel that our method for estimating Dirac eigenvalues relies strongly on this condition. Obviously, interesting split geometries $(T \neq 0)$ can only exist if $k \geq 3$, i.e., the tangent bundle splits into at least three subbundles.

Example 2.1. A metric almost contact manifold $M$ of dimension $2 n+1$ has structure group $\mathrm{U}(n)$, embedded as upper $(2 n) \times(2 n)$-matrices in $\mathrm{O}(2 n+1)$. Thus, the holonomy of a characteristic connection (if existent) is necessarily reducible, the tangent bundle $\mathcal{T} M$ splits into a $2 n$-dimensional and a onedimensional parallel distribution. This is not yet sufficient for a manifold with split holonomy; but in many cases, $\mathcal{T} M$ decomposes further with a torsion of 
split type (see Section 3 ). On the other side, a strict $G_{2}$-manifold or $\operatorname{Spin}(7)$ manifold (i.e., without further reduction to a subgroup $G \subset G_{2}, \operatorname{Spin}(7)$ ) cannot be of split holonomy, since $G_{2}$ and $\operatorname{Spin}(7)$ act by an irreducible representation.

\subsection{Dirac operators and Schrödinger-Lichnerowicz formulas}

Let us assume from now on that $M$ is also a spin manifold. Let $p_{i}$ denote the orthogonal projection from $\mathcal{T} M^{n}$ onto $\mathcal{T}_{i}$ and define the 'partial connections'

$$
\nabla_{X}^{i}:=\nabla_{p_{i}(X)}, \quad \text { hence } \nabla=\sum_{i=1}^{k} \nabla^{i}
$$

We use the same notation for their lifts to the spinor bundle $\Sigma M$. They induce the notions of 'partial Dirac operators' and 'partial spinor Laplacians' ( $\mu$ is the usual Clifford multiplication) through

$$
D_{i}:=\mu \circ \nabla^{i}, \quad D=\sum_{i=1}^{k} D_{i}, \quad \Delta^{i}:=\left(\nabla^{i}\right)^{*} \nabla^{i}, \quad \Delta=\sum_{i=1}^{k} \Delta^{i} .
$$

As long as the connection is not further specified, this is a correct definition; if $\nabla$ is chosen to be an invariant connection for a $G$ structure, i.e., a characteristic connection, the 'right' Dirac operator to consider is the characteristic Dirac operator $\not D$ associated with the connection with torsion $T / 3$. Nevertheless, we shall also use $D_{i}$ and $D$ as an intermediate tool.

At a fixed point $p \in M^{n}$ we choose orthonormal bases $e_{1}^{i}, \ldots, e_{n_{i}}^{i}$ of the distributions $\mathcal{T}_{i}(i=1, \ldots, k)$ such that $\left(\nabla_{e_{m}^{i}} e_{l}^{j}\right)_{p}=0$ for all suitable indices $i, j, m, l$. This means in particular that $\left[e_{m}^{i}, e_{l}^{j}\right]=-T\left(e_{m}^{i}, e_{l}^{j}\right)$ and $\nabla_{e_{m}^{i}}^{g} e_{m}^{i}=0$. Denoting $\nabla_{e_{m}^{i}}$ by $\nabla_{m}^{i}$, the partial Dirac and Laplace operators may then be expressed as

$$
D_{i}:=\sum_{m=1}^{n_{i}} e_{m}^{i} \nabla_{m}^{i}, \quad \Delta^{i}:=-\sum_{m=1}^{n_{i}} \nabla_{m}^{i} \nabla_{m}^{i}
$$

The divergence term of the Laplacian vanishes because of $\nabla_{e_{m}^{i}}^{g} e_{m}^{i}=0$. We compute the squares of the partial Dirac operators $D_{i}$.

Proposition 2.1. If $(M, g, \nabla)$ is a manifold with split holonomy, the partial Dirac operators $D_{i}$ satisfy the identities

$$
\left(D_{i}\right)^{2} \psi=\Delta^{i} \psi+\tilde{\sigma}_{T}^{i}+\frac{1}{4} \tau_{i} \cdot \psi
$$

where

$$
\tilde{\sigma}_{T}^{i}==\frac{1}{2} \sum_{\substack{k<l, p<q, e_{k}^{i} e_{l}^{i} e_{p} e_{q} \\ 4-\text { form }}} R\left(e_{k}^{i}, e_{l}^{i}, e_{p}, e_{q}\right) e_{k}^{i} e_{l}^{i} e_{p} e_{q}
$$

for any numbering $\left\{e_{p}\right\}_{p=1, \ldots, n}$ of the total orthonormal frame $\cup_{i=1}^{k}\left\{e_{1}^{i}, \ldots, e_{n_{i}}^{i}\right\}$. 
Proof. For the first identity, let $k$ and $l$ be indices running between 1 and $\operatorname{dim} \mathcal{T}_{i}=n_{i}$. We split the sum into terms with $k=l$ and $k \neq l$,

$$
\begin{aligned}
\left(D_{i}\right)^{2} \psi & =\sum_{k, l=1}^{n_{i}} e_{k}^{i} \nabla_{k}^{i} e_{l}^{i} \nabla_{l}^{i} \psi \\
& =-\sum_{k=1}^{n_{i}} \nabla_{k}^{i} \nabla_{k}^{i} \psi+\sum_{k \neq l} e_{k}^{i} e_{l}^{i} \nabla_{k}^{i} \nabla_{l}^{i} \psi \\
& =\Delta^{i}+\sum_{k<l} e_{k}^{i} e_{l}^{i}\left(\nabla_{k}^{i} \nabla_{l}^{i}-\nabla_{l}^{i} \nabla_{k}^{i}\right) \psi
\end{aligned}
$$

and express the second term through the curvature in the spinor bundle,

$$
\left(D_{i}\right)^{2} \psi=\Delta^{i} \psi+\sum_{k<l} e_{k}^{i} e_{l}^{i}\left[R^{\Sigma}\left(e_{k}^{i}, e_{l}^{i}\right)-\nabla_{T\left(e_{k}^{i}, e_{l}^{i}\right)}\right] \psi
$$

By our assumption of split holonomy, $T\left(e_{k}^{i}, e_{l}^{i}\right)=0$, so the corresponding term vanishes. $R^{\Sigma}$ in turn can be expressed through the curvature $R$ (see [1], [3]), and, by the curvature properties listed before, only terms with all four vectors inside $\mathcal{T}_{i}$ can occur:

$$
\begin{aligned}
\sum_{k<l} e_{k}^{i} e_{l}^{i} R^{\Sigma}\left(e_{k}^{i}, e_{l}^{i}\right) & =\frac{1}{2} \sum_{k<l} e_{k}^{i} e_{l}^{i} R\left(e_{k}^{i} \wedge e_{l}^{i}\right) \cdot \psi \\
& =\frac{1}{2} \sum_{k<l, p<q} R\left(e_{k}^{i}, e_{l}^{i}, e_{p}, e_{q}\right) e_{k}^{i} e_{l}^{i} e_{p} e_{q} \psi
\end{aligned}
$$

Note here that $e_{p}, e_{q}$ are not necessarily from $\mathcal{T}_{i}$. The summands with same indices add up to half the partial scalar curvature, while different indices yield the Clifford multiplication by the 4 -form $\tilde{\sigma}_{T}$ by $(1)$,

$$
\sum_{k<l} e_{k}^{i} e_{l}^{i} R^{\Sigma}\left(e_{k}^{i}, e_{l}^{i}\right)=\tilde{\sigma}_{T}^{i}+\frac{1}{4} \tau_{i}
$$

Recall that the characteristic Dirac operator $\not^{2}$ is linked to the Laplacian of the connection $\nabla$ through the following Schrödinger-Lichnerowicz formula ([4], [13]). Here, Scal ${ }^{g}$ and Scal denote the scalar curvatures of the Levi-Civita connection and the new connection $\nabla$, respectively, and

$$
\left.\left.\sigma_{T}:=\frac{1}{2} \sum_{k}\left(e_{k}\right\lrcorner T\right) \wedge\left(e_{k}\right\lrcorner T\right) .
$$

Theorem 2.1. For $\nabla T=0$, the spinor Laplacian $\Delta$ and the square of the Dirac operator $\not D$ are related by

(2) $\quad D^{2}=\Delta-\frac{1}{4} T^{2}+\frac{1}{4} \mathrm{Scal}^{g}+\frac{1}{8}\|T\|^{2}=\Delta^{c}+\sigma_{T}+\frac{1}{4} \mathrm{Scal}+\frac{1}{4} T^{2}$.

Then the Dirac operators $\not D$ and $D_{i}^{c}$ satisfy the following relationship: 
Proposition 2.2. If $(M, g, \nabla)$ is a manifold with split holonomy, we have

$$
\sum_{i=1}^{k} \tilde{\sigma}_{T}^{i}=\sigma_{T}
$$

which implies

$$
\sum_{i=1}^{k}\left(D_{i}\right)^{2} \psi=\Delta \psi+\sigma_{T} \psi+\frac{1}{4} \operatorname{Scal} \psi=\not D^{2} \psi-\frac{1}{4} T^{2} \psi
$$

Proof. For the identity (3), observe that $\nabla T=0$ implies $d T=2 \sigma_{T}$, hence the first Bianchi identity is reduced to

$$
\stackrel{X, Y, Z}{S} R(X, Y, Z, V)=\sigma_{T}(X, Y, Z, V) .
$$

From the symmetry property of $R(X, Z, U, V)$ with respect to $X, Y$ and $U, V$ and Lemma 2.1, it holds that $R\left(e_{m}^{i}, e_{l}^{j}\right)=0$ for $i \neq j$. Thus, we have the following equation for the 4 -form and the partial 4 -forms:

$$
\begin{aligned}
\sigma_{T} & =\frac{1}{2} \sum_{p<q, r<s} R\left(e_{p}, e_{q}, e_{r}, e_{s}\right) e_{p} e_{q} e_{r} e_{s} \\
& =\sum_{i} \frac{1}{2} \sum_{p<q, r<s, e_{p}, e_{q} \in \mathcal{T}_{i}} R\left(e_{p}, e_{q}, e_{r}, e_{s}\right) e_{p} e_{q} e_{r} e_{s}=\sum_{i} \tilde{\sigma}_{T}^{i} .
\end{aligned}
$$

The equality (4) is then a consequence of Proposition 2.1, (2) and (3).

\subsection{An adapted twistor operator}

For our eigenvalue estimate, the crucial point is to use an adapted twistor operator. Define an operator $P: \Gamma(\Sigma M) \longrightarrow \Gamma\left(T^{*} \otimes \Sigma M\right)$ by

$$
P \psi:=\nabla^{c} \psi+\sum_{i=1}^{k} \frac{1}{n_{i}} \sum_{l=1}^{n_{i}} e_{l}^{i} \otimes e_{l}^{i} \cdot D_{i}^{c} \psi .
$$

By a direct computation, one checks

$$
\|P \psi\|^{2}=\int\left\langle\left(\Delta-\sum_{i=1}^{k} \frac{1}{n_{i}}\left(D_{i}\right)^{2}\right) \psi, \psi\right\rangle d M .
$$

The crucial step is the following integral identity. Recall that the dimensions $n_{i}$ of the distributions $\mathcal{T}_{i}$ are chosen to be ordered, $n_{1} \leq n_{2} \leq \cdots \leq n_{k}$ :

Theorem 2.2. Let $(M, g, \nabla)$ be a manifold of split holonomy. Then the Dirac operator $\not D$ satisfies

$$
\begin{aligned}
\int\left(\not D^{2} \psi, \psi\right) d M= & \frac{n_{k}}{4\left(n_{k}-1\right)} \int\left(\mathrm{Scal}^{g} \psi, \psi\right) d M \\
& +\int\left(\left(\frac{n_{k}}{8\left(n_{k}-1\right)}\|T\|^{2}-\frac{1+n_{k}}{4 n_{k}-4} T^{2}\right) \psi, \psi\right) d M
\end{aligned}
$$




$$
+\frac{n_{k}}{n_{k}-1}\|P \psi\|^{2}+\frac{n_{k}}{n_{k}-1} \sum_{i=1}^{k-1}\left(\frac{1}{n_{i}}-\frac{1}{n_{k}}\right)\left\|\left(D_{i}^{c}\right)^{2} \psi\right\|^{2} .
$$

Proof. From the generalized Schrödinger-Lichnerowicz formula

$$
\not D^{2}=\Delta-\frac{1}{4} T^{2}+\frac{1}{4} \mathrm{Scal}^{g}+\frac{1}{8}\|T\|^{2} .
$$

So, we compute

$\Delta-\sum_{i=1}^{k} \frac{1}{n_{i}}\left(D_{i}\right)^{2}=\not D^{2}-\frac{1}{n_{k}}\left(D_{k}\right)^{2}-\sum_{i=1}^{k-1} \frac{1}{n_{i}}\left(D_{i}\right)^{2}-\left[-\frac{1}{4} T^{2}+\frac{1}{4} \mathrm{Scal}^{g}+\frac{1}{8}\|T\|^{2}\right]$.

By equation (4), this can be rewritten

$$
\begin{aligned}
\Delta-\sum_{i=1}^{k} \frac{1}{n_{i}}\left(D_{i}\right)^{2}= & \not^{2}-\frac{1}{n_{k}} \not D^{2}-\sum_{i=1}^{k-1}\left[\frac{1}{n_{i}}-\frac{1}{n_{k}}\right]\left(D_{i}\right)^{2} \\
& +\frac{1}{4 n_{k}} T^{2}-\left[-\frac{1}{4} T^{2}+\frac{1}{4} \mathrm{Scal}^{g}+\frac{1}{8}\|T\|^{2}\right] \\
= & {\left[\frac{n_{k}-1}{n_{k}}\right] \not D^{2}-\sum_{i=1}^{k-1}\left[\frac{1}{n_{i}}-\frac{1}{n_{k}}\right]\left(D_{i}\right)^{2} } \\
& -\frac{1}{4} \mathrm{Scal}^{g}+\left[\frac{1}{4 n_{k}}+\frac{1}{4}\right] T^{2}-\frac{1}{8}\|T\|^{2} .
\end{aligned}
$$

The identity (5) for the adapted twistor operator $P$ thus implies the desired identity.

We now recall the general Schrödinger-Lichnerowicz formula from Theorem 2.1 , which relates $\not^{2}$ and $\Delta^{c}$. Since the torsion $T$ is $\nabla$-parallel, $\Delta$ commutes with $T$, and we obtain ([5], Proposition 3.4)

$$
\not D^{2} \circ T=T \circ \not D^{2} \text {. }
$$

It is therefore possible to split the spin bundle $\Sigma M$ in the orthogonal sum of its eigenbundles for the $T$ action,

$$
\Sigma M=\bigoplus_{\mu} \Sigma_{\mu}
$$

and to consider $\not D^{2}$ on each of them, since $\nabla^{s}$ and $\not D^{2}$ both preserve this splitting. We shall henceforth denote the different eigenvalues of $T$ on $\Sigma$ by $\mu_{1}, \ldots, \mu_{l}$. This method of evaluating eigenvalues was first described in [6] (see also [19]).

Corollary 2.1. Let $\lambda$ be an eigenvalue of $D^{2}$ with an eigenspinor $\psi$ which lies in $\mu$-eigenspace of $T$. Then,

$$
\lambda\left(\left.\not D^{2}\right|_{\Sigma_{\mu}}\right) \geq \frac{n_{k}}{4\left(n_{k}-1\right)} \mathrm{Scal}_{\min }^{g}+\frac{n_{k}}{8\left(n_{k}-1\right)}\|T\|^{2}-\frac{1+n_{k}}{4\left(n_{k}-1\right)} \mu^{2}:=\beta_{\text {split }}(\mu) .
$$


The equality holds if and only if $\mathrm{Scal}^{g}$ is constant, $P(\psi)=0$ and either $n^{i}=n^{k}$ or $D_{i} \psi=0$, for all $i=1, \ldots, k$. For the smallest eigenvalue $\lambda$ of $D^{2}$ on the whole spin bundle $\Sigma M$, one thus obtains the estimate

$\lambda \geq \frac{n_{k}}{4\left(n_{k}-1\right)} \mathrm{Scal}_{\min }^{g}+\frac{n_{k}}{8\left(n_{k}-1\right)}\|T\|^{2}-\frac{1+n_{k}}{4\left(n_{k}-1\right)} \max \left(\mu_{1}^{2}, \ldots, \mu_{k}^{2}\right):=\beta_{\text {split }}$.

Proof. The inequality is a direct consequence of Theorem 2.2.

Remark 2.1. The eigenvalue estimate from [3] for reducible holonomy may not be applied in this situation. However, two other general eigenvalues may be compared to our result. Both require only the condition $\nabla T=0$, no assumption on the holonomy:

(1) In [4], it is proved that

$$
\lambda \geq \frac{1}{4} \mathrm{Scal}_{\min }^{g}+\frac{1}{8}\|T\|^{2}-\frac{1}{4} \max \left(\mu_{1}^{2}, \ldots, \mu_{k}^{2}\right)=: \beta_{\text {univ }} .
$$

This is called the universal eigenvalue estimate, because it is derived from the universal Schrödinger-Lichnerowicz formula cited in Theorem 2.1 .

(2) In the first part of [3], twistor theory is used to prove $(n:=\operatorname{dim} M)$

$\lambda \geq \frac{n}{4(n-1)} \mathrm{Scal}_{\min }^{g}+\frac{n(n-5)}{8(n-3)^{2}}\|T\|^{2}+\frac{n(4-n)}{4(n-3)^{2}} \max \left(\mu_{1}^{2}, \ldots, \mu_{k}^{2}\right)=: \beta_{\mathrm{tw}}$.

This estimate has the advantage that it yields the classical Riemannian estimate by Friedrich from [14] if $T=0$.

Remark 2.2. It is interesting to ask what the 'extreme' case would be for our new eigenvalue estimate (Corollary 2.1). If there is only parallel distribution, $\mathcal{T}=\mathcal{T}_{1}$ (i. e., $k=1$ and $n_{1}=\operatorname{dim} M$ ), the condition of split holonomy requires $T=0$ (and in particular, $\nabla T=0$ is trivially fulfilled). The estimate does then coincide with Friedrich' estimate [14], i.e., it is the best possible one.

\section{Examples}

Several examples will show that the assumption of split holonomy occurs quite naturally in the study of $G$ structures on manifolds.

Example 3.1. The twistor spaces of the only 4-dimensional compact selfdual Einstein manifolds $S^{4}$ and $\mathbb{C P}^{2}$ are the 6 -dimensional manifolds $\mathbb{C P}^{3}$ and $F(1,2)=\mathrm{U}(3) / U(1) \times U(1) \times U(1)$, the manifold of flags $l \subset v$ in $\mathbb{C}^{3}$ such that $\operatorname{dim} l=1$ and $\operatorname{dim} v=2$. It is well-known that they carry two Einstein metrics; one is Kähler (on $\mathbb{C P}^{3}$, this is exactly the Fubini-Study metric), the other is nearly Kähler. We shall henceforth be interested in their nearly Kähler structure. The characteristic connection $\nabla$ for nearly Kähler manifolds was first considered by Gray in [18] and, in this particular case, happens to coincide with the Chern connection (see the review [17] for general hermitian connections and [16] for the general description of characteristic connections on almost 
hermitian manifolds). By a theorem of Kirichenko ([8], [22]), the torsion $T$ of $\nabla$ is parallel, $\nabla T=0$, which is the first of the conditions needed for split holonomy. In [11], it was proved that the only complete, 6-dimensional, nonKähler nearly Kähler manifolds such that the characteristic connection has reduced holonomy are exactly $\mathbb{C P}^{3}$ and $F(1,2)$ (as Riemannian manifolds, both are of course irreducible). For computational details on these very interesting spaces, we refer to $[9$, Section 5.4]. In fact, one checks that in both cases, the holonomy of $\nabla$ splits the tangent space in three two-dimensional subbundles $\mathcal{T}_{i}^{2}$ (the upper index indicates the dimension)

$$
\mathcal{T} M=\mathcal{T}_{1}^{2} \oplus \mathcal{T}_{2}^{2} \oplus \mathcal{T}_{3}^{2}, \quad M=\mathbb{C P}^{3} \text { or } F(1,2)
$$

The general identities for nearly Kähler manifolds imply that $\mathrm{Scal}^{g}=30$, $\|T\|^{2}=4$ and $T$ has the eigenvalues $\mu=0$ and $\mu= \pm 2\|T\|$. Furthermore, there exist two Riemannian Killing spinors $\varphi^{ \pm}$that satisfy $\not D \varphi^{ \pm}=\mp\|T\| \varphi^{ \pm}$ [15]. To fix the ideas, in the notations of [9, Section 5.4 a)] for $M=F(1,2)$ : $\mathcal{T}_{1}=\left\langle e_{1}, e_{2}\right\rangle, \mathcal{T}_{2}=\left\langle e_{3}, e_{4}\right\rangle, \mathcal{T}_{3}=\left\langle e_{5}, e_{6}\right\rangle$, the almost complex structure and the torsion $T$ of the characteristic connection $\nabla$ are

$$
\Omega=e_{12}-e_{34}+e_{56}, \quad T=e_{245}+e_{146}-e_{236}+e_{135} .
$$

Here and in the sequel, we abbreviate exterior products $e_{i} \wedge e_{j} \wedge \cdots$ as $e_{i j \ldots}$. Thus, we are indeed in the situation of split holonomy as defined in Definition 2.1, and the eigenvalue estimate from Corollary 2.1 takes in this situation the value

$$
\lambda \geq \frac{2}{4(2-1)} \mathrm{Scal}^{g}+\frac{2}{8(2-1)}\|T\|^{2}-\frac{1+2}{4(2-1)} \max \left(0,4\|T\|^{2}\right)=4=: \beta_{\text {split }} .
$$

Thus, one sees that our estimate is optimal in this situation, since the two Killing spinors realize this lower bound. However, the result could also have been obtained directly from [4], since the bound $\beta_{\text {split }}$ coincides with the universal eigenvalue estimate $\beta_{\text {univ }}$ (see Remark 2.1). This is due to the deeper fact that the two Killing spinors are in fact $\nabla$-parallel.

Example 3.2. In [27], the author classifies 6-dimensional almost hermitian manifolds with parallel torsion by discussing the possible holonomy groups of the characteristic connection (denoted by $\nabla^{c}$ in this paper) and the normal form of the torsion. One finds that there are many more examples of manifolds with split holonomy - for example, all cases with $\operatorname{Hol}\left(\nabla^{c}\right) \subset S^{1}, T^{2}$, of which there are many interesting examples. However, it is not possible to test the eigenvalue estimate from Corollary 2.1 explicitly, since the curvature is not fixed by these data.

Example 3.3. The Stiefel manifolds

$$
M^{5}=\mathrm{SO}(4) / \mathrm{SO}(2) \text { and } M^{7}=\mathrm{SO}(5) / \mathrm{SO}(3)
$$

carry a normal homogeneous metric and a distinguished Sasaki structure; both are described in detail in [3], Example 5.1 (parameter value $t=1 / 2$ of the 
metric) and Example 5.2 (parameter value $t=1$ of the metric). Both are well-known spaces in the investigation of Riemannian spin manifolds: besides the metric that we are investigating, both carry an Einstein-Sasaki metric and, therefore, they admit two Riemannian Killing spinors ([14] for $M^{5}$, [20] for $\left.M^{7}\right)$. The characteristic connection $\nabla$ turns out to be the canonical connection of the underlying homogeneous space, hence the holonomy representation coincides with the isotropy representation (see [24]) and the torsion is automatically parallel (the space is naturally reductive). The tangent bundle splits into (again, the upper index denotes the dimension)

$$
\mathcal{T} M^{5}=\mathcal{T}_{1}^{2} \oplus \mathcal{T}_{2}^{2} \oplus \mathcal{T}_{3}^{1}, \quad \mathcal{T} M^{7}=\mathcal{T}_{1}^{3} \oplus \mathcal{T}_{2}^{3} \oplus \mathcal{T}_{3}^{1}
$$

The Sasaki direction corresponds in both cases to the one-dimensional bundle. With respect to a consecutive numbering of vectors of an orthonormal basis (this coincides with the numbering from [3]), the torsion is

$$
T_{M^{5}}=-\left(e_{135}+e_{245}\right), \quad T_{M^{7}}=-\left(e_{147}+e_{257}+e_{367}\right),
$$

so one sees that again, the manifold is spin and of split holonomy. There are two spinors that are constant under the lift of the isotropy representation, thus they define global sections and they are $\nabla$-parallel with Dirac eigenvalue $\lambda=1$. One easily checks with the geometric data given in [3] that this is equal to the bound given by all three known eigenvalue bounds,

$$
1=\beta_{\text {split }}=\beta_{\text {univ }}=\beta_{\mathrm{tw}} .
$$

This shows that our bound is, in this situation, again optimal. We suspect that these examples can be generalized to the Tanno deformation of any EinsteinSasaki manifold: they have parallel torsion and a natural splitting of the tangent bundle such that the torsion is of split type, but it seems hard to prove in general that these subbundles are indeed holonomy invariant. A description of the Tanno deformation of an Einstein-Sasaki manifold and of its characteristic connection may be found in [10].

\section{References}

[1] I. Agricola, Connections on naturally reductive spaces, their Dirac operator and homogeneous models in string theory, Comm. Math. Phys. 232 (2003), no. 3, 535-563.

2] _ The Srni lectures on non-integrable geometries with torsion, Arch. Math. (Brno) 42 (2006), 5-84.

[3] I. Agricola, J. Becker-Bender, and H. Kim, Twistorial eigenvalue estimates for generalized Dirac operators with torsion, Adv. Math. 243 (2013), 296-329.

[4] I. Agricola and Th. Friedrich, On the holonomy of connections with skew-symmetric torsion, Math. Ann. 328 (2004), no. 4, 711-748.

[5] _ The Casimir operator of a metric connection with totally skew-symmetric torsion, J. Geom. Phys. 50 (2004), no. 1-4, 188-204.

[6] I. Agricola, Th. Friedrich, and M. Kassuba, Eigenvalue estimates for Dirac operators with parallel characteristic torsion, Diff. Geom. Appl. 26 (2008), no. 6, 613-624.

[7] B. Alexandrov, The first eigenvalue of the Dirac operator on locally reducible Riemannian manifolds, J. Geom. Phys. 57 (2007), no. 2, 467-472. 
[8] B. Alexandrov, Th. Friedrich, and N. Schoemann, Almost Hermitian 6-manifolds revisited, J. Geom. Phys. 53 (2005), no. 1, 1-30.

[9] H. Baum, Th. Friedrich, R. Grunewald, and I. Kath, Twistors and Killing spinors on Riemannian manifolds, Teubner-Texte zur Mathematik, Band 124, Teubner-Verlag Stuttgart/Leipzig, 1991.

[10] J. Becker-Bender, Dirac-Operatoren und Killing-Spinoren mit Torsion, Ph.D. Thesis, University of Marburg, 2012.

[11] F. Belgun and A. Moroianu, Nearly Kähler 6-manifolds with reduced holonomy, Ann. Global Anal. Geom. 19 (2001), no. 4, 307-319.

[12] A. Besse, Einstein Manifolds, Ergebnisse der Mathematik und ihrer Grenzgebiete Bd. 10, Springer-Verlag Berlin-Heidelberg, 1987.

[13] J. M. Bismut, A local index theorem for non-Kählerian manifolds, Math. Ann. 284 (1989), no. 4, 681-699.

[14] Th. Friedrich, Der erste Eigenwert des Dirac-Operators einer kompakten, Riemannschen Mannigfaltigkeit nichtnegativer Skalarkrümmung, Math. Nachr. 97 (1980), 117146.

[15] Th. Friedrich and R. Grunewald, On the first eigenvalue of the Dirac operator on 6dimensional manifolds, Ann Global Anal. Geom. 3 (1985), no. 3, 265-273.

[16] Th. Friedrich and S. Ivanov, Almost contact manifolds, connections with torsion and parallel spinors, J. Reine Angew. Math. 559 (2003), 217-236.

[17] P. Gauduchon, Hermitian connections and Dirac operators, Boll. Un. Mat. Ital. B (7) 11 (1997), no. 2, 257-289.

[18] A. Gray, Nearly Kähler manifolds, J. Differential Geometry 4 (1970), 283-309.

[19] M. Kassuba, Eigenvalue estimates for Dirac operators in geometries with torsion, Ann. Global Anal. Geom. 37 (2010), no. 1, 33-71.

[20] I. Kath, Pseudo-Riemannian T-duals of compact Riemannian homogeneous spaces, Transform. Groups 5 (2000), no. 2, 157-179.

[21] E. C. Kim, Lower bounds of the Dirac eigenvalues on Riemannian product manifolds, math.DG/0402427.

[22] V. F. Kiricenko, K-spaces of maximal rank, Mat. Zam. 22 (1977), 465-476.

[23] S. Kobayashi and K. Nomizu, Foundations of Differential Geometry I, Wiley Classics Library, Wiley Inc., Princeton, 1991.

[24] Princeton, 1996.

[25] P.-A. Nagy, Skew-symmetric prolongations of Lie algebras and applications, J. Lie Theory 23 (2013), no. 1, 1-33.

[26] C. Olmos and S. Reggiani, The skew-torsion holonomy theorem and naturally reductive spaces, J. Reine Angew. Math. 664 (2012), 29-53.

[27] N. Schoemann, Almost Hermitian structures with parallel torsion, J. Geom. Phys. 57 (2007), no. 11, 2187-2212.

ILKA AGRICOLA

FACHBEREICh MATHEMATIK UND INFORMATIK

PhilipPS-UNIVERSitätT MARBURG

HANS-MEERWEIN-STRASSE

D-35032 MARBURG, GERMANy

E-mail address: agricola@mathematik.uni-marburg.de

HWAJEONG KIM

Department of Mathematics

HANNAM UNIVERSITY

DAEJEON 306-791, KOREA

E-mail address: hwajkim@hnu.kr 